\title{
THE FAINT AFTERGLOW AND HOST GALAXY OF THE SHORT-HARD GRB 060121
}

\author{
A. J. Levan, ${ }^{1,2}$ N. R. Tanvir, ${ }^{1,3}$ A. S. Fruchter, ${ }^{4}$ E. Rol,${ }^{3}$ J. P. U. Fynbo, ${ }^{5}$ J. Hjorth, ${ }^{5}$ G. Williams, ${ }^{6}$ E. Bergeron, ${ }^{4}$ \\ D. Bersier, ${ }^{7}$ M. Bremer, ${ }^{8}$ T. Grav, ${ }^{9}$ P. Jakobsson,${ }^{5}$ K. Nilsson,${ }^{10}$ E. Olszewski, ${ }^{6}$ R. S. Priddey, ${ }^{1}$ D. Rafferty, ${ }^{11}$ And \\ J. RHOADS ${ }^{12}$ \\ Received 2006 March 11; accepted 2006 July 13; published 2006 August 18
}

\begin{abstract}
We present optical and X-ray observations of the afterglow and host galaxy of the short-hard GRB 060121. The faint $R$-band afterglow is seen to decline as $t^{-0.66 \pm 0.09}$ while the X-ray falls as $t^{-1.18 \pm 0.04}$, indicating the presence of the cooling break between the two frequencies. However, the $R$-band afterglow is very faint compared to the predicted extrapolation of the X-ray afterglow to the optical regime (specifically, $\beta_{\mathrm{Ox}} \sim 0.2$ ), while the $K$-band is consistent with this extrapolation $\left(\beta_{\mathrm{Kx}} \sim 0.6\right)$, demonstrating suppression of the optical flux. Late-time $H S T$ observations place stringent limits on the afterglow $R$-band flux, implying a break in the $R$-band light curve. They also show that the burst occurred at the edge of a faint red galaxy, presumably the host, which most likely lies at a significantly higher redshift than the previous optically identified short-duration bursts. Several neighboring galaxies also have very red colors that are similarly suggestive of higher redshift. The least extreme explanation for the faintness and color of the burst is that it occurred at moderately high redshift and was significantly obscured; however, it is also possible that it lies at $z>4.5$, in which case the faintness of the $R$-band afterglow could be attributed to the Lyman break. We discuss the implications that either scenario would have for the nature of the progenitors of short bursts.
\end{abstract}

Subject heading: gamma rays: bursts

\section{INTRODUCTION}

The inquiry into the nature of short-duration $\gamma$-ray bursts (GRBs) has seen rapid progress following the discovery of the first X-ray (Gehrels et al. 2005; Bloom et al. 2006), optical (e.g., Hjorth et al. 2005a; Fox et al. 2005; Berger et al. 2005), and radio (Soderberg et al. 2006) afterglows. These observations show that, typically, short bursts have fainter afterglows than those of long-duration bursts, lie at lower redshift (usually $z<1$ ), and are associated with galaxies of all types, including those with no sign of ongoing star formation (Gehrels et al. 2005; Berger et al. 2005). The latter fact indicates a significant delay between the formation of the progenitor stars and the creation of the GRB. Indeed, in no case has any sign of supernova emission been seen (e.g., Hjorth et al. 2005b). These characteristics contrast markedly with those of long bursts that originate in star-forming host galaxies (e.g., Fruchter et al. 1999; Christensen et al. 2004) at higher redshift (Jakobsson et

${ }^{1}$ Centre for Astrophysics Research, University of Hertfordshire, Hatfield, AL10 9AB, UK.

${ }^{2}$ Kavli Institute for Theoretical Physics, University of California, Santa Barbara, CA 93106.

${ }^{3}$ Department of Physics and Astronomy, University of Leicester, University Road, Leicester LE1 7RH, UK.

${ }^{4}$ Space Telescope Science Institute, 3700 San Martin Drive, Baltimore, MD 21218.

${ }^{5}$ Dark Cosmology Centre, Niels Bohr Institute, University of Copenhagen, Juliane Maries Vej 30, 2100 Copenhagen, Denmark.

${ }^{6}$ Steward Observatory, University of Arizona, 933 North Cherry Avenue, Tucson, AZ 85721.

${ }^{7}$ Astrophysics Research Institute, Liverpool John Moores University, Twelve Quays House, Egerton Wharf, Birkenhead CH41 1LD, UK.

${ }^{8}$ Department of Physics, Bristol University, H. H. Wills Laboratory, Tyndall Avenue, Bristol BS8 1TL, UK.

${ }^{9}$ Institute for Astronomy, 2680 Woodlawn Drive, Honolulu, HI 96822.

${ }^{10}$ Tuorla Observatory, Väisäläntie 20, FIN-21500 Piikkiö, Finland.

${ }^{11}$ Department of Physics and Astronomy, Ohio University, Athens, $\mathrm{OH}$ 45701.

${ }^{12}$ Department of Physics and Astronomy, Arizona State University, P.O. Box 871504, Tempe, AZ 85287. al. 2006) and are associated with the core collapse of massive stars (e.g., Hjorth et al. 2003). The preferred explanation for short bursts has thus become that the majority of them come from either neutron star-neutron star (NS-NS) or neutron starblack hole (NS-BH) mergers (e.g., Eichler et al. 1989; Davies et al. 2005; Lee et al. 2005), although other mechanisms that produce GRBs in populations of all ages (e.g., Usov 1992; Dermer \& Atoyan 2006; Levan et al. 2006b) remain possible given the paucity of observational constraints so far.

Here we present optical and X-ray observations of shorthard GRB 060121. The afterglow of GRB 060121 is approximately a magnitude fainter than the other examples at similar times. In previous cases a bright host galaxy was readily identified under the optical transient; however, in the case of GRB 060121 this is not the case. In fact, deep Hubble Space Telescope (HST) observations, reported below, were necessary to locate a faint, red galaxy at the location of the optical afterglow.

\section{OBSERVATIONS}

GRB 060121 was detected by the High Energy Transient Explorer (HETE-2) on 2006 January 21, 22:24:54.5 UT. It was identified as a short and spectrally hard burst with a peak energy of $120 \pm 7 \mathrm{keV}$ (see Donaghy et al. 2006 for further details). Swift performed observations of GRB 060121, beginning at 2006 January 22 01:21:37 UT. X-Ray Telescope (XRT) observations revealed a relatively bright X-ray afterglow (Mangano et al. 2006).

Optical observations of GRB 060121 began $2 \mathrm{hr}$ postburst and were taken with the Nordic Optical Telescope (NOT), the $2.3 \mathrm{~m}$ Bok Telescope of the Steward Observatory, and the Wisconsin-Indiana-Yale-NOAO telescope (WIYN). A log of the observations is shown in Table 1, and all were reduced in the standard fashion within IRAF.

Inspection of the position of the X-ray afterglow of GRB 060121 revealed a faint optical afterglow (Levan et al. 2006a; also reported by Malesani et al. 2006). Its location was deter- 
TABLE 1

PhOtOMETRIC OBSERVATIONS OF GRB 060121

\begin{tabular}{|c|c|c|c|c|c|}
\hline Date & UT & $\begin{array}{c}\Delta t \\
(\mathrm{hr})\end{array}$ & Telescope & $\begin{array}{l}\text { Band } \\
\text { (AB) }\end{array}$ & Magnitude \\
\hline \multirow{7}{*}{2006 Jan $22 \ldots \ldots$} & $00: 23: 53$ & 1.98 & NOT & $R$ & $22.65 \pm 0.21$ \\
\hline & 01:00:21 & 2.59 & NOT & $I$ & $21.96 \pm 0.30$ \\
\hline & 04:02:45 & 5.63 & Bok $2.3 \mathrm{~m}$ & $R$ & $23.79 \pm 0.19$ \\
\hline & 04:52:06 & 6.48 & Bok $2.3 \mathrm{~m}$ & $B$ & $>24.0$ \\
\hline & $05: 40: 29$ & 7.25 & Bok $2.3 \mathrm{~m}$ & $R$ & $23.72 \pm 0.15$ \\
\hline & 09:41:39 & 11.27 & Bok $2.3 \mathrm{~m}$ & $R$ & $23.44 \pm 0.25$ \\
\hline & $11: 32: 15$ & 13.12 & WIYN & $R$ & $23.75 \pm 0.20$ \\
\hline 2006 Jan 23 & $07: 58: 22$ & 33.56 & WIYN & $R$ & $24.91 \pm 0.16$ \\
\hline
\end{tabular}

NOTES. - Photometry of the afterglow of GRB 060121 obtained at the NOT, the Bok $2.3 \mathrm{~m}+90$ prime imager (Williams et al. 2004), and the WIYN. Magnitudes are in the AB system and are not corrected for the small Galactic extinction $\left(A_{R}=0.044\right)$

mined by performing astrometry between the SDSS and our first WIYN image, yielding R.A. $=09^{\mathrm{h}} 09^{\mathrm{m}} 51^{\mathrm{s}} 99$, decl. $=$ $45^{\circ} 39^{\prime} 45^{\prime \prime}$.6. We performed photometry of the afterglow using an aperture with radius equal to the FWHM of the images. Photometric calibration was based on the SDSS observations of the field, converting between SDSS and Cousins filters using the transformations of Jester et al. (2005). The resulting light curve is shown in Figure 1. The temporal decay is best fit with $\alpha=-0.66 \pm 0.09\left(\alpha\right.$ is defined as $\left.F_{t} \propto t^{\alpha}\right)$. However, the $\chi^{2} /$ dof $=11.27 / 4$ is relatively poor for this fit, largely due to an apparent plateau/flare between $\sim 5$ and $11 \mathrm{hr}$ after the burst. Given the small number of data points, it is not possible to characterize this feature in detail. Later IR observations have been reported by de Ugarte Postigo et al. (2006) and are roughly simultaneous with our final WIYN observation. Given their reported magnitude of $K_{s} \sim 20$, the afterglow color was very red $(R-K \approx 5$ ), corresponding to a spectral slope (defined as $\left.F_{\nu} \propto \nu^{\beta}\right)$ of $\beta_{\mathrm{RK}}=-2.7$.

Swift XRT data were reduced using xrtpipeline. The light curve and spectrum were extracted via xselect. Data from the first 3 orbits were filtered on grade 0 to counter pileup, and later orbits used default filtering. A circular extraction region of radius of $71^{\prime \prime}$ was employed for the first 3 orbits, while a $47^{\prime \prime}$ radius circle was used for later orbits. The background was estimated using extraction regions around the source and has been dynamically subtracted from the light curve and spectrum. For both the light curve and the spectrum, 20 counts were grouped together per bin.

The X-ray light curve (Fig. 1, bottom) has been modeled with a single power law with decay index $\alpha=-1.18 \pm$ $0.04\left(\chi^{2} / \mathrm{dof}=28.52 / 27\right)$. The data are consistent with monotonic evolution to $\sim 10$ days postburst, ruling out a jet break occurring before this time. Unfortunately, no X-ray data exist for the period covering the possible optical plateau. Thus we cannot rule out a plateau in the X-ray data, in which the underlying decay was unaffected. However, as the X-ray light curve continues as a single power law, energy injection as seen in GRB 051221 (Soderberg et al. 2006) can be ruled out.

The data used for the spectrum were obtained from the first 3 orbits. The spectrum can be fit with an absorbed power law, with $\Gamma=2.33_{-0.23}^{+0.26}, N_{\mathrm{H}}=1.31_{-0.24}^{+0.46} \times 10^{21} \mathrm{~cm}^{-2}\left(\chi^{2} /\right.$ dof $=$ 8.11/14). The Galactic absorption in this direction is $1.7 \times$ $10^{20} \mathrm{~cm}^{-2}$ (Dickey \& Lockman 1990). Some interdependence is seen between $N_{\mathrm{H}}$ and $\Gamma$; however, this is insufficient to explain the observed excess $N_{\mathrm{H}}$. We therefore conclude that GRB 060121 exhibits significant X-ray absorption above the Galactic value. This absorption estimate assumes zero redshift. At higher $z$ the rest-frame absorption may be substantially larger.

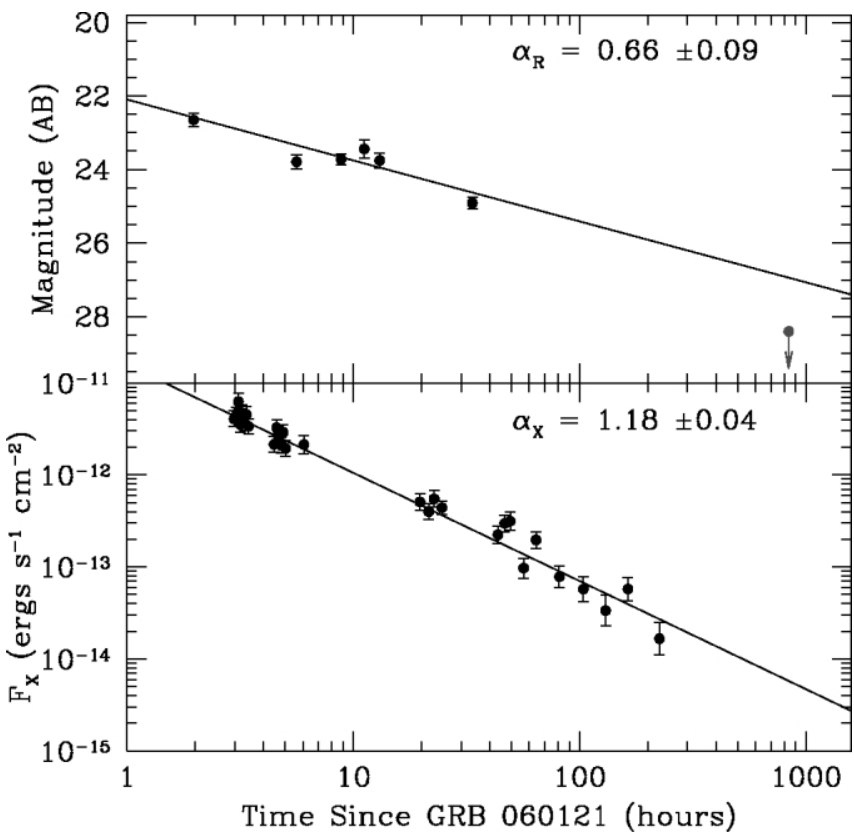

FIG. 1.- $R$-band (top) and X-ray $(0.2-10 \mathrm{keV}$; bottom) light curves of GRB 060121, fitted with single-power-law decay indices as shown and discussed in $\S 2.1$. The F606W upper limit has been converted to an $R(\mathrm{AB})$ limit (28.2) assuming the observed $R-K$ spectral slope for the afterglow, although in practice the small wavelength shifts between the two filters mean that the result is not highly sensitive to the assumed spectrum.

\subsection{HST Observations}

GRB 060121 was observed with $H S T$ on 2006 February 27. Images were obtained with NICMOS/F160W (Fig. 2, right) and ACS/WFC/F606W (Fig. 2, left). We performed astrometry relative to our ground-based images using 10 point sources in common, obtaining the location of the afterglow accurate to $0.1(1 \sigma)$. At this position there is no obvious afterglow in either the F160W or F606W images. However, there is a faint galaxy with $\mathrm{F} 606 \mathrm{~W}(\mathrm{AB})=27.0 \pm 0.3$ and $\mathrm{F} 160 \mathrm{~W}(\mathrm{AB})=$ $24.5 \pm 0.2$. Extrapolating to $R$ and $H$ using the measured spectral slopes between the two bands yields magnitudes (in the Vega system) of $R=26.6 \pm 0.3$ and $H=22.8 \pm 0.2$. The centroid of the galaxy in the F160W image lies offset from that in the F606W image by $\sim 0.3$, indicating either a color gradient in the host or possibly a contribution from the afterglow in the IR. Limiting magnitudes were derived by measuring the $\mathrm{S} / \mathrm{N}$ for a number of sources and extrapolating to a $3 \sigma$ limit. The resulting limit for the afterglow in F606W is 28.3, $\mathrm{F} 160 \mathrm{~W}=25.0$. Note that for F160W this is shallower than the frame limit since the we cannot unambiguously distinguish between host light or faint underlying afterglow in the NICMOS images. In the optical the slope from the final WIYN epoch and those obtained with $H S T$ is found to be $\alpha>1.7$, indicating a steepening decline. In addition, there are five extremely red objects (EROs) in the field that are exceptionally faint (and of low surface brightness) or undetected with ACS, but relatively bright in F160W. Some of these are very red, $\mathrm{F} 606 \mathrm{~W}(\mathrm{AB})-\mathrm{F} 160 \mathrm{~W}(\mathrm{AB})>4$, and fall among the reddest galaxies observed in GOODS with $R-K_{s}>4.35$ (AB), a population comprising only $\sim 20 \%$ of the total ERO population (Moustakas et al. 2004). Galaxies this red have an areal density on the sky of $0.41 \pm 0.05 \mathrm{arcmin}^{-2}$ to a depth of $K \approx 20$ (Gilbank et al. 2003), and thus in our observed field of only 0.5 $\operatorname{arcmin}^{2}$ they are overdense by a factor of $\sim 20$, although it 

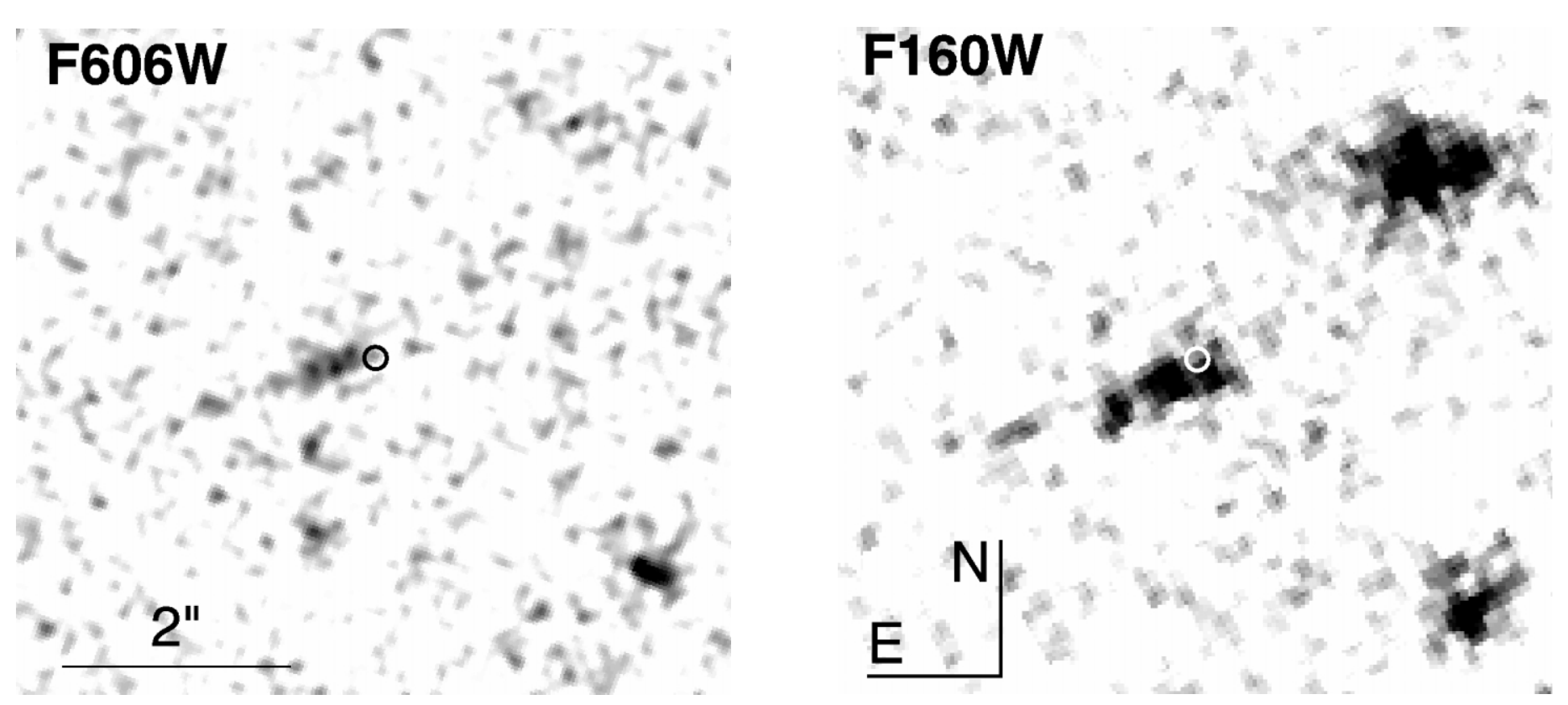

FIG. 2.- HST observations of GRB 060121 with ACS/F606W (left; smoothed by a Gaussian of the same width as a PSF to enhance faint features) and NICMOS/ F160W (right). In each case the location of the burst is marked with a white circle. As can be seen, the burst lies on an IR brighter region that may indicate either a color gradient or a continuing contribution from the afterglow in the IR. An ERO can be seen to the northwest of the host in NICMOS.

should be noted that EROs tend to be highly clustered. These objects may either represent a highly reddened population at moderate redshift $(\sim 2)$ or could lie at very high redshift $(z \sim$ $5)$, although in the latter case they would be significantly brighter than typical Lyman break galaxies at $z \sim 5$ (e.g., Lehnert \& Bremer 2003).

\section{AFTERGLOW PROPERTIES}

GRB 060121 exhibits a bright X-ray afterglow, but is very faint in the optical, at $R \sim 23$ only $2 \mathrm{hr}$ after the burst. This is somewhat fainter than previously reported optical afterglows of short bursts (e.g., Hjorth et al. 2005a; Soderberg et al. 2006). The optical to X-ray spectral slope is flatter than expected for the fireball model $\left(\beta_{\mathrm{Ox}}=0.2\right)$; in other words the optical flux is significantly lower than expected based on the extrapolation of the X-ray, thus rendering GRB 060121 a dark burst (Jakobsson et al. 2004; Rol et al. 2005). However, the value of $\beta_{\mathrm{KX}}=0.6$ is broadly consistent with the extrapolation of the $\mathrm{X}$-rays to the $K$ band assuming that the cooling break lies between the two frequencies. Indeed, the X-ray and optical light curves shown in Figure 1 demonstrate a slower decay in the optical than in the X-ray and are also consistent with the presence of the cooling break. These parameters (with exception of the $\beta_{\mathrm{OX}}$, discussed below) are consistent with those expected from a $p \sim 2.2$ fireball model (e.g., Panaitescu \& Kumar 2000).

The extrapolation of the optical light curve to late times is above the limit obtained by HST and indicates that a temporal break has occurred. There is no break in the X-ray light curve to 10 days postburst; however, optical monitoring ceased after 1.5 days. If the break in the $R$ band is due to the jet break, then it would have occurred $>10$ days postburst and the required slope would be $\alpha>1.7$. If this break were the cooling break, then it could have occurred in the $R$ band and been unobserved in the X-ray. If it occurred at the time of the final $R$-band observations, then the late-time slope is only constrained to be $\alpha>1.1$, consistent with the X-ray slope, and thus with the motion of the cooling break. However, the value of $\beta_{\mathrm{KX}} \sim$ 0.6 indicates that the cooling break lies close to the $\mathrm{X}$-ray band at $\sim 30 \mathrm{hr}$, and thus is unlikely to reach the $R$ band until very late times. We believe that the most likely cause of the steepening of the optical slope is the jet break.

A natural explanation of the SED is that the $R$-band afterglow is extinguished due to the presence of dust in the host galaxy. This would be supported by the red $R-K$ color and the high column measured from the X-ray spectrum. The X-rays and (to a lesser extent) $K$ band are largely unaffected by the presence of dust, while the $R$ band (or rest-frame $B$ or UV, depending on $z$ ) exhibits the strongest signature of extinction. The location of GRB 060121 in a possibly edge-on galaxy, in which significant dust extinction could occur through the disk, lends some support to this theory. Although the GRB does not lie near the centroid of the galaxy, it does apparently lie along the major axis of the galaxy, consistent with an origin in the disk.

An alternative explanation for the faint $R$-band afterglow is that the burst lies at significantly higher redshift $(z \sim 5)$. In this case the low value of $\beta_{\text {ox }}$ would be produced by the Ly $\alpha$ break lying within the $R$ band, while the $K$ band would be unaffected and would lie on the extrapolation of the X-ray afterglow (as is observed). However, in this case we might expect to observe a stronger break between the $R$ and $I$ bands than is actually seen. For example, GRB 000131 at $z=4.5$ had $R-I=$ 1.2 (Andersen et al. 2000) and GRB 050814 at $z=5.3 \mathrm{had}$ $R-I \sim 2.5$ (Jakobsson et al. 2006), while the measured color of GRB 060121 (extrapolating $I$ to the same epoch as our first $R$-band observation) is $R-I=0.90 \pm 0.36$. This value has a large error due to the low signal-to-noise ratios in our images and in fact is consistent with the color of GRB 000131 (although also with a typical $\nu^{-1}$ spectrum). A higher redshift comparable to that of GRB 050814 is apparently ruled out, although $z \sim 4.5$ certainly remains plausible. Indeed, de Ugarte Postigo et al. (2006) obtain similar estimates of the redshift via fitting the multiwavelength SED of the afterglow. Further 
observations of the host galaxy will be helpful in distinguishing between a strong break due to Ly $\alpha$ and gradual reddening.

\section{NATURE AND ENERGETICS}

GRB 060121 was a relatively bright burst. If it lay at $z \approx$ 1 (a lower redshift would be hard to reconcile with the faintness of the host galaxy) then its isotropic energy release would be $E_{\text {iso }} \sim 1.3 \times 10^{52}$, while at higher redshift $(z=4.5)$ it would be $E_{\text {iso }} \sim 2.0 \times 10^{53} \mathrm{ergs}$ (both values in the $2-400 \mathrm{keV}$ range). Either of these energies would be markedly higher than previously observed short-duration bursts. Assuming that the jet break occurred after $\sim 10$ days (but before the HST observations), this would correspond to jet opening angles of $\gtrsim 10^{\circ}$ and $5^{\circ}$, and thus true energies of at least $E_{\gamma}=2 \times 10^{50}$ and $6.4 \times 10^{50}$ ergs, respectively. The moderately late jet break is consistent with that seen in other short bursts (e.g., Watson et al. 2006), while the true energies $\left(E_{\gamma}\right)$ are roughly an order of magnitude larger than seen in GRB 051221 (Burrows et al. 2006), the most luminous short burst to date with a definite redshift.

The currently most popular model for (most) short GRBs is that they originate from compact binary (NS-NS or NS-BH) mergers. At formation a NS receives a significant natal kick (e.g., Arzoumanian et al. 2002); thus, NS-NS binaries may leave their birthplaces with velocities of several hundred $\mathrm{km} \mathrm{s}^{-1}$. Their merger time is governed by the gravitational radiation timescale and can span a wide range of times from $10^{6}$ to $10^{10} \mathrm{yr}$ (e.g., Burgay et al. 2003). However, even at the shorter end of this range the NS-NS binary would have traveled $>100 \mathrm{pc}$ from its birth site, and out of the region of star formation in which it formed. It is, however, unlikely that the faintness of the afterglow in the $R$ band is due to its being in the IGM; both the $K$ band and X-rays were reasonably bright.

Finally it is also important to consider whether GRB 060121 could in fact be due to a collapsar. Although its spectrum is moderately hard the duration of $\sim 2 \mathrm{~s}$ puts it within a region of the BATSE hardness-duration plot that contains an admixture of long-, short-, and possibly intermediate-duration bursts (Horváth et al. 2006). Indeed, some classical long-duration bursts have rest-frame durations of $<2$ s (e.g., GRB 000301C [Jensen et al. 2001], GRB/XRF 050416 [Sakamoto et al. 2006], and GRB 060206 [Fynbo et al. 2006]). However, considering a range of properties Donaghy et al. (2006) conclude that GRB 060121 belongs to the short-hard burst population.

\section{CONCLUSIONS}

We have presented deep optical and X-ray observations of the short-hard GRB 060121. These observations demonstrate that both the afterglow and host galaxy of GRB 060121 are significantly fainter and redder than previously optically detected short bursts.

We believe the most likely explanation of its properties is that GRB 060121 lies at higher redshift than previously observed short bursts, $z>2$, and furthermore is probably extinguished. If this is a compact binary merger then significant dust extinction is surprising since it would be expected that the binaries should receive significant natal kicks. Thus, in this case either the binary received very little kick or (by chance) its line of sight passed through intervening dust.

Interestingly, GRB 060121 lies rather closer, in the hardnessduration sense, to the typical short burst as seen by BATSE than the other well-localized short bursts studied to date. GRBs 050709 and 050724 , in particular, were all very much at the spectrally soft end of that distribution (Hjorth et al. 2005a). This raises the question as to whether a significant proportion of the bursts classified by BATSE as short could in fact be a much higher redshift population than those seen recently by Swift and HETE-2. If so, then the width of the luminosity function of short bursts would be much wider than has previously been considered (e.g., Piran \& Guetta 2006), with consequent implications for progenitor models.

We thank the referee for a constructive report, and J. Heidt and C. Villforth for assistance at the NOT. A. J. L., N. R. T., and E. R. are supported by PPARC. The Dark Cosmology Centre is funded by the DNRF. Based on observations made with HST obtained at STScI, under NASA contract NAS526555 (program 10870). Partly supported by the NSF under grant PHY 99-07949.

\section{REFERENCES}

Andersen, M. I., et al. 2000, A\&A, 364, L54

Arzoumanian, Z., Chernoff, D. F., \& Cordes, J. M. 2002, ApJ, 568, 289

Berger, E., et al. 2005, Nature, 438, 988

Bloom, J. S., et al. 2006, ApJ, 638, 354

Burgay, M., et al. 2003, Nature, 426, 531

Burrows, D. N., et al. 2006, ApJ, submitted (astro-ph/0604320)

Christensen, L., Hjorth, J., \& Gorosabel, J. 2004, A\&A, 425, 913

Davies, M. B., Levan, A. J., \& King, A. R. 2005, MNRAS, 356, 54

Dermer, C. D., \& Atoyan, A. 2006, ApJ, 643, L13

de Ugarte Postigo, A., et al. 2006, ApJL, submitted (astro-ph/0605516)

Dickey, J. M., \& Lockman, F. J. 1990, ARA\&A, 28, 215

Donaghy, T. Q., et al. 2006, ApJ, submitted (astro-ph/0605570)

Eichler, D., Livio, M., Piran, T., \& Schramm, D. N. 1989, Nature, 340, 126

Fox, D. B., et al. 2005, Nature, 437, 845

Fruchter, A. S., et al. 1999, ApJ, 519, L13

Fynbo, J. P. U., et al. 2006, A\&A, 451, L47

Gehrels, N., et al. 2005, Nature, 437, 851

Gilbank, D. G., Smail, I., Ivison, R. J., \& Packham, C. 2003, MNRAS, 346, 1125

Hjorth, J., et al. 2003, Nature, 423, 847

2005a, Nature, 437, 859

2005b, ApJ, 630, L117

Horváth, I., Balázs, L. G., Bagoly, Z., Ryde, F., \& Mészáros, A. 2006, A\&A, 447, 23

Jakobsson, P., Hjorth, J., Fynbo, J. P. U., Watson, D., Pedersen, K., Björnsson, G., \& Gorosabel, J. 2004, ApJ, 617, L21
Jakobsson, P., et al. 2006, A\&A, 447, 897

Jensen, B. L., et al. 2001, A\&A, 370, 909

Jester, S., et al. 2005, AJ, 130, 873

Lee, W. H., Ramirez-Ruiz, E., \& Granot, J. 2005, ApJ, 630, L165

Lehnert, M. D., \& Bremer, M. 2003, ApJ, 593, 630

Levan, A. J., Tanvir, N. R., Fynbo, J., Hjorth, J., Fruchter, A., Grav, T., \& Nilsson, K. 2006a, GCN Circ. 4562, http://gcn.gsfc.nasa.gov/gcn/gcn3/ 4562.gen 3

Levan, A. J., et al. 2006b, MNRAS, 368, L1

Malesani, D., et al. 2006, GCN Circ. 4561, http://gcn.gsfc.nasa.gov/gcn/gcn3/ 4561.gcn 3

Mangano, V., La Parola, V., Mineo, T., Tagliaferri, G., Romano, P., O’Brien, P., \& Burrows, D. N. 2006, GCN Circ. 4560, http://gcn.gsfc.nasa.gov/gen/ gen3/4560.gcn3

Moustakas, L. A., et al. 2004, ApJ, 600, L131

Panaitescu, A., \& Kumar, P. 2000, ApJ, 543, 66

Piran, T., \& Guetta, D. 2006, in AIP Conf. Proc. 838, Gamma-Ray Bursts in the Swift Era, ed. S. S. Holt, N. Gehrels, \& J. A. Nousek (Melville: AIP), 58

Rol, E., Wijers, R. A. M. J., Kouveliotou, C., Kaper, L., \& Kaneko, Y. 2005, ApJ, 624, 868

Sakamoto, T., et al. 2006, ApJ, 636, L73

Soderberg, A. M., et al. 2006, ApJ, submitted (astro-ph/0601455)

Usov, V. V. 1992, Nature, 357, 472

Watson, D., et al. 2006, A\&A, 454, L123

Williams, G. G., et al. 2004, Proc. SPIE, 5492, 787 